\title{
On robust lot sizing problems with storage deterioration, with applications to heat and power cogeneration
}

\author{
S. Coniglio ${ }^{1}$, A. M.C.A. Koster ${ }^{2}$, and N. Spiekermann ${ }^{2}$ \\ ${ }^{1}$ Department of Mathematical Sciences, University of Southampton \\ University Road, Southampton, SO17 1BJ, United Kingdom \\ s.coniglio@soton.ac.uk \\ ${ }^{2}$ Lehrstuhl II für Mathematik, RWTH Aachen University \\ Pontdriesch 14-16, 52062, Aachen, Germany \\ \{koster, spiekermann\}@math2.rwth-aachen.de
}

\begin{abstract}
We consider a variant of the single item lot sizing problem where the product, when stored, suffers from a proportional loss, and in which the product demand is affected by uncertainty. This setting is particularly relevant in the energy sector, where the demands must be satisfied in a timely manner and storage losses are, often, unavoidable. We propose a two-stage robust optimization approach to tackle the problem with second stage storage variables. We first show that, in the case of uncertain demands, the robust problem can be solved as an instance of the deterministic one. We then address an application of robust lot sizing arising in the context of heat and power cogeneration and show that, even in this case, we can solve the problem as an instance of the deterministic lot sizing problem. Computational experiments are reported and illustrated.
\end{abstract}

\section{Introduction}

Lot Sizing (LS) is the fundamental problem of a large part of modern production planning systems. In its basic version, given a demand for a single good over a finite time horizon, the problem calls for a feasible production plan which minimizes storage, production, and setup costs, also guaranteeing that certain lower and upper bounds on both the production and the amount of good that is stored at each point in time are met.

In the paper, we focus on a generalized version of lot sizing where the product suffers from proportional losses when stored and the objective function is not necessarily a linear function of the production variables. We also assume uncertain product demands and, consequently, we will tackle the problem from a robust optimization perspective. This suits the case of many applications in the energy sector where the product demands of, typically, heat or power, are often not known in advance, especially when the decision maker has to commit to a production plan some time before it becomes operational. 
In the paper, we first show that the robust counterpart of this generalized version of the lot sizing problem can be solved as a special instance of its deterministic counterpart with suitably defined demands and storage upper bounds. We next investigate an application of this result to a production planning problem arising in the context of Combined Heat and Power Production (CHPP), which we also show to be solvable as a special instance of the deterministic (generalized) lot sizing problem.

The paper is organized as follows. In Section 2, we report on some previous work on lot sizing and introduce some relevant robust optimization concepts. Our main contribution, the reduction of the version of robust lot sizing problem that we consider to the deterministic one, is outlined in Section 3. Section 4 illustrates the application to heat and power cogeneration, while Section 5 collects some computational results and observations. Section 6 concludes the paper with some final comments.

\section{Previous work}

In this section, we give a brief account of some relevant work on different versions of the lot sizing problem, also encompassing the uncertain case.

\subsection{Deterministic case}

Many variants of the deterministic lot sizing problem have been studied in the literature. For an extensive account of the most relevant work, we refer the reader to the monography [PW06]. The problem is known to be in $\mathcal{P}$ for the case with linear costs, complete conservation (i.e., no losses in the stored product), zero storage lower bounds, nonzero time dependent storage upper bounds, and no production bounds, as shown by Atamtürk and Küçükyavuz in [AK08]. A similar result holds for nonnegative and nondecreasing concave cost functions, complete conservation, production bounds constant over time, and unrestricted storage, as shown by Hellion, Mangione, and Penz in [HMP12]. For a polynomial time algorithm for the case with storage losses and nondecreasing concave costs, but no storage or production bounds, see the work of Hsu [Hsu00]. As to $\mathcal{N} \mathcal{P}$-hard cases, Florian, Lenstra, and Kan in [FLK80] provide a number of examples. These include the case of linear as well as fixed production costs, no inventory costs, no storage bounds, no lower production bounds, but nonconstant production upper bounds.

\subsection{Uncertain case}

Classical approaches to handle uncertainties in lot sizing are, historically, stochastic in nature, dating back as early as 1960 [Sca60]. The idea is to first assign a probability distribution to the uncertain demand and, then, to solve the problem by looking for a solution of minimum expected cost. Unfortunately, as pointed out in [LS05], even when the distribution is estimated within sufficient precision 
from historical data, such methods can yield solutions which, when implemented with the demand that realizes in practice, can be substantially more costly than those that were predicted with the stochastic approach. Moreover, and regardless of the accuracy of the estimation, these techniques are, in many cases, intrinsically doomed to suffer from the curse of dimensionality [BT06], as they usually require a computing time which is, at least, linear in the size of the (discrete) probability space, which is typically exponential in the size of the instance.

A different option, arguably more affordable from a computational standpoint, is of resorting to a robust optimization approach, thus looking for solutions which are feasible for any realization of the uncertain demand belonging to a given uncertainty set, and which also minimize the worst case cost. Two seminal papers in this direction are those of Bertsimas and Thiele [BT04,BT06], who consider the uncapacitated lot sizing problem with backlogging ${ }^{3}$ and fixed costs, with demands subject to a so-called $\Gamma$-robustness model of uncertainty ${ }^{4}$. Among other results, they show that the $\Gamma$-robust counterpart of the variant of lot sizing they consider can be solved as a version of the deterministic problem with modified demands, also for the case where production bounds are in place.

We remark that, for the result by Bertsimas and Thiele to hold, bounds on the storage cannot be enforced. This is an issue in the energy sector, where backlogging is not tolerable as the demand of energy, be it heat or electrical power, must be satisfied when issued.

\section{Robust lot sizing under demand uncertainty}

In this section, we first introduce the generalized variant of lot sizing that we will address. After presenting its robust counterpart, we will then illustrate how to reduce this robust problem to a special version of the deterministic one and draw some computational complexity considerations.

\subsection{Lot sizing with deterioration and storage bounds}

Consider a single product and a time horizon $T=\{1, \ldots, n\}$. For each time step $t \in T$, let $d_{t} \geq 0$ be the product demand, $q_{t} \geq 0$ the production variable, $z_{t} \in\{0,1\}$ an indicator variable equal to 1 if $q_{t}>0$, and $u_{t} \geq 0$ a variable corresponding to the value of the storage at the end of time period $t$. Let also $u_{0} \geq 0$ be the initial storage value. We assume time dependent bounds $\underline{Q}_{t} \leq$ $q_{t} \leq \bar{Q}_{t}$ on the production and $\underline{U}_{t} \leq u_{t} \leq \bar{U}_{t}$ on the storage. The generalized

\footnotetext{
${ }^{3}$ In case of backlogging, shortages in the inventory are allowed - or, said differently, unmet demand can be postponed, at a cost, to the future.

${ }^{4}$ Originally proposed in [BS03,BS04], the idea of $\Gamma$-robustness is of assuming that the uncertain parameters (demands for LS) belong to symmetric intervals and that, for an integer $\Gamma$, the total number of uncertain demands which deviate from their nominal value to either of the extremes of their intervals is bounded by $\Gamma$ in any solution and constraint of the problem.
} 
variant of lot sizing that we consider can be cast as the following Mixed-Integer Linear Programming (MILP) formulation:

$$
\begin{aligned}
& (\mathrm{LS}-\mathrm{DET}) \min f(q, z)+\sum_{t \in T} c_{t}^{i} u_{t} \\
& \text { s.t. } \quad \alpha_{t} u_{t-1}+q_{t}=u_{t}+d_{t} \quad \forall t \in T \\
& U_{t} \leq u_{t} \leq \overline{U_{t}} \quad \forall t \in T \\
& z_{t} \underline{Q_{t}} \leq q_{t} \leq z_{t} \overline{Q_{t}} \quad \forall t \in T \\
& z_{t} \in\{0,1\} \quad \forall t \in T \\
& q_{t} \geq 0 \quad \forall t \in T \\
& u_{t} \geq 0 \quad \forall t \in T .
\end{aligned}
$$

The generalization goes along two directions. First, we assume that the Objective (1a) is the sum of a (general) function $f(q, z)$ of variables $q, z$ and a linear one of $u, \sum_{t \in T} c_{t}^{i} u_{t}$, with inventory $\operatorname{costs} c_{t}^{i}$. The classical case is obtained for $f(q, z)=\sum_{t \in T}\left(a_{t} q_{t}+b_{t} z_{t}\right)$, where $a_{t}$ and $b_{t}$ are, resp., the unit production cost and the setup cost at time $t \in T$. Secondly, we assume that, at the end of each time period $t \in T$, a fraction $\left(1-\alpha_{t}\right)$ of the stored product is lost, as determined by the (possibly time dependent) conservation factor $\alpha_{t} \in(0,1]$.

We remark that, similarly to the classical case where $\alpha_{t}=1$ for all $t \in T$, in every feasible solution $(q, z, u)$ of LS-DET, $u_{t}$ is uniquely determined as a function of $q_{t}, u_{t-1}$, and $d_{t}$. Indeed, from Constraint (1b), we deduce, by substitution from $t=1$ to $t=|T|$ :

$$
u_{t}:=\left(\prod_{k=1}^{t} \alpha_{k}\right) u_{0}+\sum_{i=1}^{t}\left(\prod_{k=i+1}^{t} \alpha_{k}\right)\left(q_{i}-d_{i}\right) \quad \forall t \in T .
$$

Note that Equation (2) is causal, i.e., that the storage at time $t$ only depends on the demand (and production) at times $1, \ldots, t-1$. From the equation, it follows that a pair $(q, z)$ suffices to fully characterize a solution to LS-DET, as the (unique) value of the missing vector $u$ can be calculated a posteriori from Equation (2). We call a pair $(q, z)$ satisfying Constraints (1d)-(1f) a production plan and denote by $\mathcal{I}_{\text {LS-DET }}(q, z)=(q, z, u)$, with $u$ as in Equation (2), its induced solution to LS-DET.

\section{$3.2 \quad$ Uncertain demands}

Assuming that the uncertain demand vector $d$ takes values in the uncertainty set $D$, the robust counterpart of LS-DET, i.e., a version of the problem where we look for a solution $(q, z, u)$ which is feasible for all realizations $d \in D$, has to be a solution to:

$$
\begin{aligned}
(\text { LS-ROB }) \quad \min & (1 \mathrm{a}) \\
\text { s.t. } \quad & \alpha_{t} u_{t-1}+q_{t}=u_{t}+d_{t} \quad \forall t \in T, d \in D \\
& (1 \mathrm{c})-(1 \mathrm{~g}),
\end{aligned}
$$


where the original Constraints (1b) (which are called Constraint (3b) here) are enforced for all realizations $d_{t} \in D$. As we will better explain in the following, we remark that, in this case, all the variables are first stage variables, i.e., they are required to take a single value which is independent of the realization of $d$.

For any nontrivial $D$, the following holds:

Proposition 1. If $\exists\left\{d^{1}, d^{2}\right\} \subseteq D$ with $d^{1} \neq d^{2}$, then LS-ROB is infeasible.

Proof. For any value of $q, d$, Constraints (3b) induce the linear system $A u=$ $q-d+e_{1} \alpha_{1} u_{0}$, where $A$ is a full rank matrix with the all-one vector as main diagonal and the vector $-\alpha$ as the diagonal below it. Assuming that LS-ROB is feasible, we have $\exists u: A u=q-d^{1}+e_{1} \alpha_{1} u_{0}$ and $A u=q-d^{2}+e_{1} \alpha_{1} u_{0}$. This implies $A^{-1}\left(q-d^{1}+e_{1} \alpha_{1} u_{0}\right)=A^{-1}\left(q-d^{2}+e_{1} \alpha_{1} u_{0}\right)$, that is, $d^{1}=d^{2}$, a contradiction.

It is thus natural, as well as very reasonable in practice, to assume that the value of the storage $u_{t}$ at time $t \in T$ can be adapted as a function $u_{t}(d)$ of the demand $d \in D$ which has realized up to time $t-1$, i.e., that $u$ is a second stage variable. A direct formulation for the robust counterpart of LS-DET with a second stage $u$ is thus:

$$
\begin{aligned}
& \text { (LS-ROB2) } \min f(q, z)+\eta \\
& \text { s.t. } \quad \alpha_{t} u_{t-1}(d)+q_{t}=u_{t}(d)+d_{t} \quad \forall t \in T, d \in D \\
& \underline{U_{t}} \leq u_{t}(d) \leq \overline{U_{t}} \quad \forall t \in T, d \in D \\
& \eta \geq \sum_{t \in T} c_{t}^{i} u_{t}(d) \quad \forall d \in D \\
& \text { (1d)-(1f) } \\
& u_{t}(d) \geq 0 \\
& \eta \geq 0 \text {. }
\end{aligned}
$$

The newly introduced variable $\eta$ accounts for the worst case storage cost over all $d \in D$ (a so-called, partial, epigraph reformulation). Assuming, as it is the case for a discrete scenario approach, a finite $D$, LS-ROB2 calls for a vector $u(d) \geq 0$ for each $d \in D$ which satisfies Constraints (4b)-(4d). Clearly, LSROB2 can be solved directly by employing a mixed-integer linear programming solver, although at the cost of expressing Constraints (4b)-(4d) and (4f) $|D|$ times.

\subsection{Solving LS-ROB2 as an instance of LS-DET}

We now present a more efficient way to solve LS-ROB2 as an instance of LS-DET with suitably chosen demand and storage upper bound vectors $d$ and $\bar{U}$.

As for LS-DET, a production plan $(q, z)$ is required to satisfy Constraints (1d)(1f), which are condensed, in LS-ROB2, in Constraint (4e). Its induced solution 
$\mathcal{I}_{\mathrm{LS}-\mathrm{ROB} 2}(q, z)=(q, z, u(d), \eta)$ to LS-ROB2 can be defined, w.l.o.g., as:

$$
\begin{aligned}
u_{t}(d) & :=\left(\prod_{k=1}^{t} \alpha_{k}\right) u_{0}+\sum_{i=1}^{t}\left(\prod_{k=i+1}^{t} \alpha_{k}\right)\left(q_{i}-d_{i}\right) \quad \forall t \in T, d \in D \\
\eta & :=\max _{d \in D}\left\{\sum_{t \in T} c_{t}^{i} u_{t}(d)\right\} .
\end{aligned}
$$

Differently from the case of LS-DET, another induced solution can be constructed by selecting any $\eta$ satisfying $\eta>\max _{d \in D}\left\{\sum_{t \in T} c_{t}^{i} u_{t}(d)\right\}$. Clearly though, such solution cannot be optimal.

Our main result follows:

Theorem 1. For an uncertainty set $D$ over which a linear function can be optimized in polynomial time, LS-ROB2 can be polynomially reduced (w.r.t. production plans) to an instance of LS-DET with $d=d^{\prime}$ and $\bar{U}=\bar{U}^{\prime}$ thus defined:

$$
\begin{array}{rlrl}
d_{t}^{\prime} & :=\max _{d \in D}\left\{d_{t}-\sum_{i=1}^{t-1}\left(\prod_{k=i+1}^{t} \alpha_{k}\right)\left(d_{i}^{\prime}-d_{i}\right)\right\} & & \forall t \in T \\
\bar{U}_{t}^{\prime}:=\bar{U}_{t}-\Delta_{t} & \forall t \in T \\
\Delta_{t}:=\max _{d \in D}\left\{\sum_{i=1}^{t}\left(\prod_{k=i+1}^{t} \alpha_{k}\right)\left(d_{i}^{\prime}-d_{i}\right)\right\} & \forall t \in T .
\end{array}
$$

Proof. First, note that the values for $d_{t}^{\prime}$ and $\bar{U}_{t}^{\prime}$ can be computed iteratively, from $t=1$ to $t=|T|$, in polynomial time due to the assumptions on $D$. For a given production plan $(q, z)$, adopting $d=d^{\prime}$ and $\bar{U}=\bar{U}^{\prime}$, we show that $\mathcal{I}_{\mathrm{LS}-\mathrm{ROB} 2}(q, z)=$ $(q, z, u(d), \eta)$ is feasible for LS-ROB2 if and only if $\mathcal{I}_{\text {LS-DET }}(q, z)=(q, z, u)$ is feasible for LS-DET. We deduce the following:

$$
\begin{array}{rlrl}
d_{t}^{\prime} & \geq 0 & & \forall t \in T \\
u_{t} & =\min _{d \in D}\left\{u_{t}(d)\right\} & & \forall t \in T \\
u_{t}+\Delta_{t} & =\max _{d \in D}\left\{u_{t}(d)\right\} & & \forall t \in T \\
\eta & =\sum_{t \in T} c_{t}^{i} u_{t}+\underbrace{\max _{d \in D}\left\{\sum_{t \in T} c_{t}^{i} \sum_{i=1}^{t} \prod_{k=i}^{t} \alpha_{k}\left(d_{i}^{\prime}-d_{i}\right)\right\}}_{\text {const }} &
\end{array}
$$

For the derivations, which we omit due to space reasons, we refer the reader to the Online Appendix [CKS16]. We are to show that Constraints (4c) are satisfied by $u(d)$ if and only if Constraints (1c) are satisfied by $u$ (as all the other constraints are satisfied by definition of production plan). This is shown by observing that, for all $t \in T$, the following holds true:

$$
\begin{aligned}
& \underline{U}_{t} \leq u_{t}(d) \quad \forall d \in D \Leftrightarrow \underline{U}_{t} \leq \min _{d \in D}\left\{u_{t}(d)\right\} \Leftrightarrow \underline{U}_{t} \leq u_{t} \\
& u_{t}(d) \leq \bar{U}_{t} \forall d \in D \Leftrightarrow \max _{d \in D}\left\{u_{t}(d)\right\} \leq \bar{U}_{t} \Leftrightarrow u_{t}+\Delta_{t} \leq \bar{U}_{t} \Leftrightarrow u_{t} \leq \bar{U}_{t}^{\prime} .
\end{aligned}
$$


Since Objectives (4a) and (1a) are equal up to a constant additive term, i.e.:

$$
f(q, z)+\eta=f(q, z)+\sum_{t \in T} c_{t}^{i} u_{t}+\text { const }
$$

we deduce that a production plan is optimal for LS-ROB2 if and only if it is optimal for LS-DET.

Intuitively, the newly defined demand $d_{t}^{\prime}$ induces a lower bound on the product which has to be available at time $t \in T$, thus ensuring that every demand $d \in D$ can be met. The value $\Delta$ reduces the storage upper bound of the transformed problem to prevent that, when a large production is realized but, suddenly, a large deficit in demand occurs (an event which would result in an overflow of storage), the actual storage upper bound $\bar{U}_{t}$ is not exceeded.

We remark that the assumptions in Theorem 1 subsume the cases of many widely employed robustness models, including polyhedral uncertainty sets (such as the $\Gamma$-robustness one), discrete scenario uncertainty sets, or ellipsoidal uncertainty sets, such as those used in [BTEGN09].

From a computational complexity perspective, the following holds:

Corollary 1. Given an uncertainty set $D$ over which a linear function can be optimized in polynomial time and suitable assumptions on the givens, LS-ROB2 is in $\mathcal{P}$ (resp., $\mathcal{N} \mathcal{P}$-hard) if and only if the corresponding version of LS-DET is in $\mathcal{P}$ (resp., $\mathcal{N} \mathcal{P}$-hard).

Proof. To polynomially reduce LS-ROB2 to LS-DET, use Lemma 1. For the polynomial reduction from LS-DET to LS-ROB2, consider that every deterministic problem can be regarded as a robust optimization problem.

Note that, as a consequence of Corollary 1, LS-ROB2 is in $\mathcal{P}$ for all the polynomially solvable cases of LS-DET that we reported in Section 2 for which the algorithm allows for time dependent upper bounds $\bar{U}$ on $u$. This is, for instance, the case of the problem studied in [HMP12].

We conclude by noting that our result can be slightly extended as follows:

Remark 1. Theorem 1 and Corollary 1 still hold if we introduce additional constraints on $z$ and $q$ or assumptions on the givens (except for $d$ and $\bar{U}$ ). They are also valid for $\overline{U_{t}}=\infty$ and for not necessarily nonnegative demands $d$.

\section{Application to heat and power cogeneration}

In this section, we consider an application of the previous results to the case of Combined Heat and Power Production (CHPP). CHPP plants are production units in which the heat that is generated when cooling down the plant is extracted and, at least partially, utilized for heating purposes. The units are equipped with a storage tank where the heat in excess can be, temporarily, stored, subject to constant proportional losses due to dissipation effects over time. 
From a production planning perspective, two products and two demands are present: one of heat and one of power. Power in excess or defect w.r.t. the given demand can be sold or bought from the power marked. Storage is costless, while a cost is incurred for fuel consumption. In principle, we can outline three sources of uncertainty: heat demands, power demands, and power market prices. Among the three, heat demands are, arguably, the most critical. This is because badly estimated heat demands can lead to infeasible production plans where the storage bounds are violated. Differently, badly estimated power demands or market prices can only introduce an extra cost in the objective function. For this reason, in the following we will focus solely on uncertain heat demands.

\subsection{Problem formulation}

We adopt the same variables as in LS-DET, with $q, u$, and $d^{h}$ representing the amount of heat which is, resp., produced, stored, and required as a demand. For power, we introduce a second production variable $p_{t}$, indicating the amount of power that is generated at time $t \in T$, two market variables, $p^{b}$ and $p^{s}$, representing the amount of power that is, at each point in time, bought and sold, and a demand vector $d^{p}$. Let $c^{p}$ be the vector of market prices for both buying and selling a MWh of power and let $c^{f}$ be the vector of fuel prices. Fuel consumption at time $t$, as denoted by the variable $f_{t}$, is modeled as the linear function $s q_{t}+h z_{t}$, where $h z_{t}$ is a constant term corresponding to the activation of the CHPP unit. We assume that heat and power are produced with a fixed proportion $\rho \in(0,1)$ and that $\alpha$ is constant over $T$.

Let $D^{h}$ be the uncertainty set for the heat demands. As for LS-ROB2, we assume second stage storage variables $u\left(d^{h}\right)$ as a function of the uncertain heat demand $d^{h} \in D^{h}$. We introduce the following robust two-stage MILP formulation:

$$
\begin{array}{lll}
\text { (CHPP-ROB2) } \min & \sum_{t \in T}\left(c_{t}^{p}\left(p_{t}^{b}-p_{t}^{s}\right)+c_{t}^{f} f_{t}\right) & \\
\text { s.t. } & \alpha u_{t-1}\left(d^{h}\right)+q_{t}=u_{t}\left(d^{h}\right)+d_{t}^{h} & \\
& p_{t}^{p}+p_{t}^{b}=d_{t}^{p}+p_{t}^{s} & \forall t \in T, d^{h} \in D^{h} \\
& \underline{U} \leq u_{t}\left(d^{h}\right) \leq \bar{U} & \forall t \in T, d^{h} \in D^{h} \\
& z_{t} \underline{Q} \leq q_{t} \leq z_{t} \bar{Q} & \forall t \in T \\
& f_{t}=s q_{t}+h z_{t} & \forall t \in T \\
& p_{t}^{p}=\rho q_{t} & \forall t \in T \\
& q_{t}, f_{t}, p_{t}^{p}, p_{t}^{b}, p_{t}^{s} \geq 0 & \forall t \in T \\
& u_{t}\left(d^{h}\right) \geq 0 & \forall t \in T, d^{h} \in D^{h} \\
& z_{t} \in\{0,1\} & \forall t \in T .
\end{array}
$$


Given a production plan $(q, z)$, its induced solution to CHPP-ROB2, i.e., $\mathcal{I}_{\mathrm{CHPP}-\mathrm{ROB} 2}(q, z)=\left(q, z, f, p^{p}, p^{b}, p^{s}, u\left(d^{h}\right)\right)$, is defined as:

$$
\begin{aligned}
u_{t}\left(d^{h}\right) & =\alpha^{t} u_{0}+\sum_{i=1}^{t} \alpha^{t-i}\left(q_{i}-d_{i}^{h}\right) & & \forall t \in T \\
f_{t} & =s q_{t}+h z_{t} & & \forall t \in T \\
p_{t}^{p} & =\rho q_{t} & & \forall t \in T \\
p_{t}^{b} & =\max \left\{d_{t}^{p}-p_{t}^{p}, 0\right\} & & \\
p_{t}^{s} & =\max \left\{p_{t}^{p}-d_{t}^{p}, 0\right\} . & &
\end{aligned}
$$

The following holds:

Proposition 2. CHPP-ROB2 can be solved as an instance of LS-DET.

Proof. By substitution from Constraint (10g), Constraints (10c) become, for all $t \in T$ :

$$
p_{t}^{b}-p_{t}^{s}=d_{t}^{p}-\rho q_{t}
$$

which, after substitution, together with Constraint (10f), in Objective (10a), yield:

$$
\sum_{t \in T}\left(c_{t}^{p}\left(d_{t}^{p}-\rho q_{t}\right)+c_{t}^{f}\left(s q_{t}+h z_{t}\right)\right)=\sum_{t \in T}\left(\left(c_{t}^{f} s-c_{t}^{p} \rho\right) q_{t}+c_{t}^{f} h z_{t}\right)+\underbrace{\sum_{t \in T} c_{t}^{p} d_{t}^{p}}_{\text {const }} .
$$

By setting:

$$
\begin{aligned}
f(q, z) & :=\sum_{t \in T}\left(\left(c_{t}^{f} s-c_{t}^{p} \rho\right) q_{t}+c_{t}^{f} h z_{t}\right) \\
\alpha_{t} & :=\alpha \quad \forall t \in T \\
c_{t}^{i} & :=0 \quad \forall t \in T
\end{aligned}
$$

and dropping the constant term, we obtain an instance of LS-ROB2. The corresponding instance of LS-DET is obtained by applying Corollary 1.

Unfortunately, we are not aware of any specialized algorithm capable of solving LS-DET with $\alpha_{t}<1$ in combination with (constant) lower and upper production bounds. In spite of this, in the next section we will rely on the transformation into LS-DET to solve CHPP-ROB2 via mixed-integer linear programming techniques in a much shorter amount of computing time than when tackling it directly in its original form. 


\section{Computational results}

We report on a set of computational experiments on a CHPP-ROB2 problem originating within the project Robuste Optimierung der Stromversorgungsysteme (Robust Optimization of Power Supply Systems), funded by the German Bundesministerium für Wirtschaft und Energie (Federal Ministry for Economic Affairs and Energy, BMWi).

We consider a dataset of 232 days, spanning a period of two years (with some missing months). Market prices for the power market are taken from EPEX STOP (the European Power Exchange). The power demand is taken from historical data for the whole country of Germany, downscaled to 50000 households, while the heat demand data is taken from a portion of Frankfurt (of around 50000 households). We assume $s=1.51 \mathrm{EUR} / \mathrm{MWh}, h=5.43 \mathrm{EUR} / \mathrm{MWh}$, and $\rho=0.4$. The bounds are set to $\underline{U}=0 \mathrm{MWh}, \bar{U}=120 \mathrm{MWh}, \underline{Q}=37.5 \mathrm{MWh}$, $\bar{Q}=125 \mathrm{MWh}$. We also set $u_{0}=36 \mathrm{MWh}$.

We adopt a discrete scenario uncertainty set $D^{h}$, built from a heat demand forecast provided by our industrial partner ProCom GmbH. The first scenario of $D^{h}$ is the original forecast for the current day, as produced by ProCom. It is generated in a two-stage fashion, with an autoregressive component and a neural network one, with temperature and calendar events as main influence factors. We then single out the 50 days from the set of historical time series where the corresponding pair of demand and forecast is closest in L1 norm. After computing the forecast error between the two, we create a scenario where such error is added to the forecast demand of the current day (for which the problem is being solved). This way we, intuitively, "learn" the forecast error from historical data and apply it to the current forecast, creating 50 additional scenarios. The general idea is that the forecast error follows certain patterns, so that it is more likely that combinations of the historical forecast errors will also apply to the error of the current day for which CHPP-ROB2 is being solved.

The experiments are run on an Intel i7-3770 $3.40 \mathrm{GHz}$ machine with $32 \mathrm{~GB}$ RAM using CPLEX 12.6 and AMPL as modeling language. We consider four settings, with a time horizon of, resp., 24, 48, 72, and 96 hours. The total time in seconds to solve all the instances, as well as the standard deviation, are reported in the following table. In it, as well as in the charts that will follow, CHPP-ROB2 accounts for the problem when solved via the original Formulation (10a)-(10j), whereas LS-DET corresponds the problem being solving via Formulation (1a)(1g) after begin transformed, by applying Theorem 1, into an instance of the deterministic lot sizing problem. Proportional speedup factors are also reported.

\begin{tabular}{c|rr|rr||cc} 
& \multicolumn{2}{|c|}{ CHPP-ROB2 } & \multicolumn{2}{c||}{ LS-DET } & \multicolumn{2}{c}{ Speedup } \\
\hline horizon & totTime stdev & totTime stdev & totTime stdev \\
\hline $24 \mathrm{~h}$ & 37.05 & 0.05 & 7.96 & 0.01 & 4.65 & 5.00 \\
$48 \mathrm{~h}$ & 140.01 & 0.17 & 16.32 & 0.03 & 8.58 & 5.67 \\
$72 \mathrm{~h}$ & 323.95 & 0.47 & 34.77 & 0.15 & 9.32 & 3.13 \\
$96 \mathrm{~h}$ & 805.86 & 2.65 & 64.49 & 0.42 & 12.50 & 6.31
\end{tabular}


As the table illustrates, the improvement in computing time achieved when solving the problem as an instance of LS-DET, rather than in its original form, steeply increases with size of the instances. From an average speedup of 4.65 times for the $24 \mathrm{~h}$ instances, we register one of 8.58 times for the $48 \mathrm{~h}$ instances, one of 9.32 times for the $72 \mathrm{~h}$ instances, and one of 12.5 times for the $96 \mathrm{~h}$ instances. On average, the speedup is of 7.69 times. This illustrates that, even when adopted in a mixed-integer linear programming setting, the transformation proposed in Theorem 1 allows for a substantial reduction in the computing times. The table also shows that the computing time, if seen as a stochastic process, becomes much more stable when Theorem 1 is employed. Indeed, with its application, we observe a reduction in the standard deviation ranging between 3.13 times for the $72 \mathrm{~h}$ instances to 6.31 times for the $96 \mathrm{~h}$ ones, with an average reduction of 5.03 times. A visual depiction is reported in Figure 1.

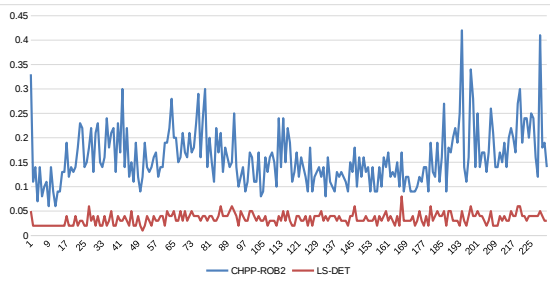

(a)

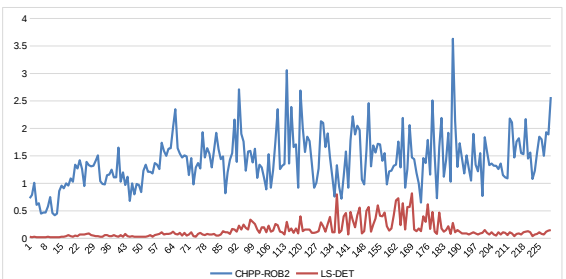

(c)

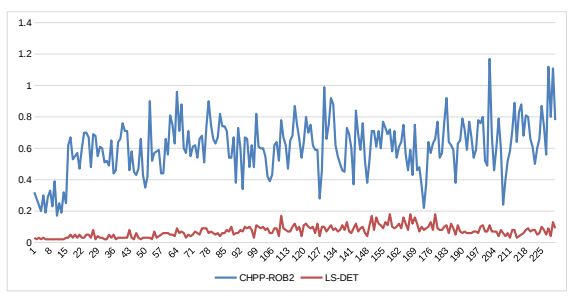

(b)

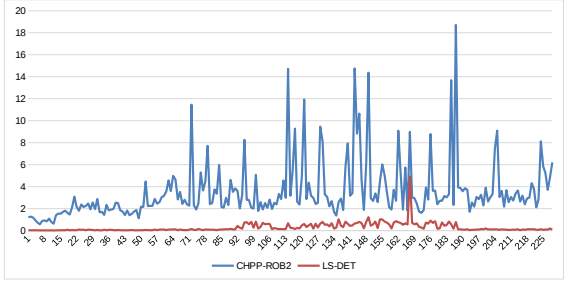

(d)

Fig. 1. Computing times when solving CHPP-ROB2 in its original form vs. those when solving it as an instance of LS-DET via the transformation outlined in Theorem 1. Each instance is composed of: (a) 24 hours, (b) 48 hours, (c) 72 hours, (d) 96 hours.

\section{Concluding remarks}

We have considered a generalized variant of lot sizing with proportional storage losses and a nonlinear objective function, showing how, for the case of uncertain demands, the problem can be solved as a special instance of the deterministic one via a polynomial time transformation. We have then considered an application to heat and power cogeneration systems, showing that, when uncertain demands 
are considered, even that problem can be tackled as a special instance of lot sizing. Computational experiments have shown that our transformation allows us to solve the problem in a much shorter computing time even when using a general purpose mixed-integer linear programming solver.

Acknowledgement This work is supported by the German Federal Ministry for Economic Affairs and Energy, BMWi, grant 03ET7528B.

\section{References}

[AK08] A. Atamtürk and S. Küçükyavuz. An algorithm for lot sizing with inventory bounds and fixed costs. Operations Research Letters, 36(3):297 - 299, 2008.

[BS03] D. Bertsimas and M. Sim. Robust discrete optimization and network flows. Mathematical programming, 98(1-3):49-71, 2003.

[BS04] D. Bertsimas and M. Sim. The price of robustness. Operations research, 52(1):35-53, 2004.

[BT04] B. Bertsimas and A. Thiele. A robust optimization approach to supply chain management. Integer programming and combinatorial optimization, pages 145-156, 2004.

[BT06] D. Bertsimas and A. Thiele. A robust optimization approach to inventory theory. Operations Research, 54(1):150-168, 2006.

[BTEGN09] Aharon Ben-Tal, Laurent El Ghaoui, and Arkadi Nemirovski. Robust optimization. Princeton University Press, 2009.

[CKS16] S. Coniglio, A. M.C.A. Koster, and N. Spiekermann. Online appendix. 2016. https://www.dropbox.com/s/16a33b756uxr25j/appendix.pdf?dl=0.

[FLK80] M. Florian, J. K. Lenstra, and A. H. G. Rinnooy Kan. Deterministic production planning: Algorithms and complexity. Management Science, 26(7):669-679, 1980.

[HMP12] B. Hellion, F. Mangione, and B. Penz. A polynomial time algorithm to solve the single-item capacitated lot sizing problem with minimum order quantities and concave costs. European Journal of Operational Research, 222(1):10 - 16, 2012.

[Hsu00] V.N. Hsu. Dynamic economic lot size model with perishable inventory. Management Science, 46(8):1159-1169, 2000.

[LS05] L.H. Liyanage and J.G. Shanthikumar. A practical inventory control policy using operational statistics. Operations Research Letters, 33(4):341-348, 2005.

[PW06] Y. Pochet and L.A. Wolsey. Production planning by mixed integer programming. Springer, 2006.

[Sca60] H. Scarft. The optimally of $(S, s)$ policies in the dynamic inventory problem. In Mathemtical Methods in the Social Sciences, volume 1, page 196. Stanford University Press, 1960. 